\title{
Polyline Fitting of Planar Points under Min-Sum Criteria
}

\author{
Boris Aronov* $^{*}$ Tetsuo Asano ${ }^{\dagger}$ Naoki Katoh ${ }^{\ddagger}$ Kurt Mehlhorn ${ }^{\S}$ \\ Takeshi Tokuyama
}

\begin{abstract}
Fitting a curve of a certain type to a given set of points in the plane is a basic problem in statistics and has numerous applications. We consider fitting a polyline with $k$ joints under the min-sum criteria with respect to $L_{1}$ - and $L_{2}$-metrics, which are more appropriate measures than uniform and Hausdorff metrics in statistical context. We present efficient algorithms for the 1-joint versions of the problem and fully polynomial-time approximation schemes for the general $k$-joint versions.
\end{abstract}

\section{Introduction}

Curve fitting aims to approximate a given set of points in the plane by a curve of a certain type. This is a fundamental problem in statistics, and has numerous applications. In particular, it is a basic operation in regression analysis. Linear regression approximates a point set by a line, while non-linear regression approximates it by a non-linear function from a given family.

In this paper, we consider the case where the points are fitted by a polygonal curve (polyline) with $k$ joints, see Figure 1 . This is often referred to as polygonal approximation or polygonal fitting problem. It is used widely. For example, it is commonly employed in scientific and business analysis to represent a data set by a polyline with a small number of joints. The best representation is the polyline minimizing the error of approximation. Error is either defined as the maximum (vertical) distance of any input point from the polyline (min-max-optimization) or the sum of vertical distances (min-sum-approximation).

Min-max-approximation by a polyline is well studied. In one popular formulation one minimizes the maximum of the vertical distance (called the uniform metric or Chebyschev error function) from the points to the curve. Hakimi and Schmeichel gave a $O\left(n^{2} \log n\right)$ time algorithm for this problem [11]; the time complexity was later improved to $O\left(n^{2}\right)$ [27] and then to $O(n \log n)$ [10]. Another popular approach is to minimize the Hausdorff measure that is the maximum of the Euclidean distances between the points and the output curve. This problem can also be solved in polynomial time [23]. These problems are closely related to curve simplification, in which the input is a polyline with $n$ edges rather than a set of $n$ points; this question arises in geographic information systems (see the survey [28]) and has received much attention in computational geometry $[5,12,15,22]$.

The minimize-the-maximum ( $\min$-max) formulation is useful in pattern recognition applications. However, in applications to statistics, its serious deficiency is its extreme sensitivity to

\footnotetext{
*Polytechnic University, Brooklyn, NY 11201, USA; http://cis.poly.edu/ a aronov. Supported in part by NSF ITR Grant CCR-00-81964. Part of the work was carried out while B.A. was visiting JAIST, Universitat Politècnica de Catalunya, and MPII.

${ }^{\dagger}$ Japan Advanced Institute of Science and Technology, Tatsunokuchi, 923-1292, Japan; t-asano@jaist.ac.jp. Partially supported by the Ministry of Education, Science, Sports and Culture, Grant-in-Aid for Scientific Research (B).

${ }^{\ddagger}$ Kyoto University, Kyoto, 606-8501, Japan; naoki@archi.kyoto-u.ac.jp.

$\S$ Max-Planck-Institut für Informatik, D-66123, Saarbrücken, Germany; mehlhorn@mpi-sb.mpg.de.

ITohoku University, Sendai, 980-8579, Japan; tokuyama@dais.is.tohoku.ac.jp.
} 


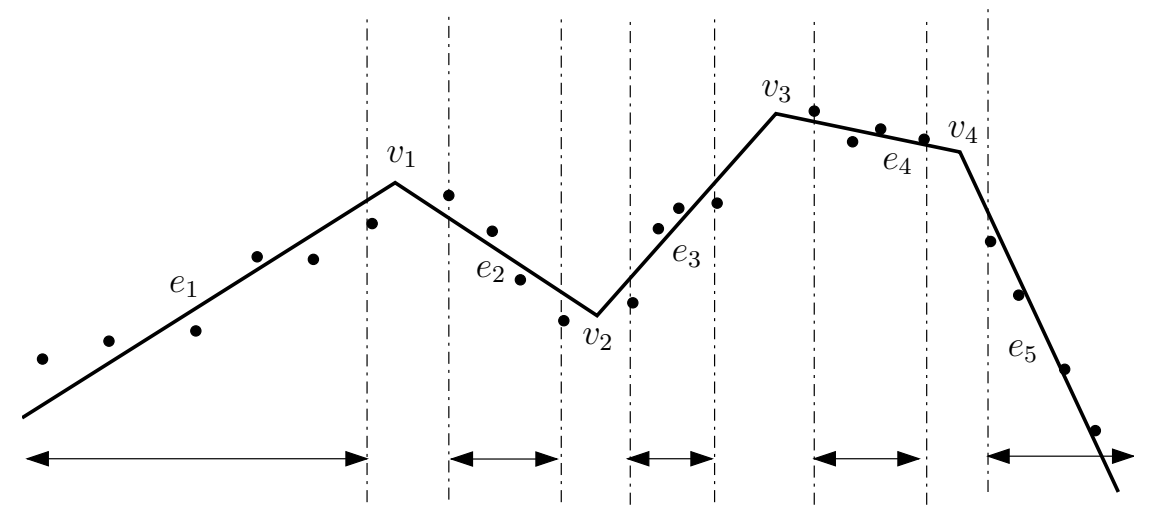

Figure 1: A 4-joint polyline fitting a set of points.

the presence and location of outliers. Even a single outlier can drastically change the output, while outliers, real or imagined, are common in statistical data. For this reason, minimize-thesum-of-errors (min-sum) methods are considerably more popular in statistics: The most basic one is the least-squares method that minimizes the sum of the squares of vertical distances between the input points and the output curve. In this paper, we call it the $L_{2}$-fitting problem (the term least-squares fitting is commonly used as well), and regression line in statistics usually refers to the $L_{2}$-fitting line. If the output curve is either a straight line or a low-degree algebraic curve, it is quite easy to compute the optimal $L_{2}$ fitting. Another criterion is $L_{1}$-minimization, in which we minimize the sum of vertical distances from the candidate curve to the points being fitted. $L_{1}$ fitting is more resilient to outliers than $L_{2}$ fitting; however, it is usually more expensive (or complicated) to compute the optimal solution. For linear regression, the $L_{1}$-optimal line can be computed in linear time [13], but it requires sophisticated computational techniques. Several other formulations have been proposed for further reducing the effect of outliers on the linear regression. Repeated median regression is a well-known example, and efficient solutions are known for several other criteria [16]. TAKESHI SAYS: Inserted a reference suggested by a referee. BORIS SAYS: ok, slightly reworded Linear regression considering Euclidean or Manhattan distance between points and the line has also been studied [21].

In this paper, we focus on the $L_{1^{-}}$and $L_{2}$-fitting problems when the desired curve is a $k$ joint polyline; in other words, it is a continuous piecewise-linear $x$-monotone curve with $k+1$ linear components. We assume that a coordinate system is fixed, and the input points are sorted with respect to their $x$-coordinate values. To the authors' knowledge, the computational complexity of the optimal $k$-joint problem under either of these minimization criteria has not been previously investigated. More specifically, it seems that an efficient solution of the $L_{1}$-fitting problem extending the result of Imai et al. [13] is theoretically challenging even for the 1-joint problem.

In this paper, we begin by considering the 1-joint problem. We give algorithms of complexity $O(n)$ and $\tilde{O}\left(n^{4 / 3}\right)$ time for the $L_{2}$ and $L_{1}$ criteria, respectively. ${ }^{1}$ The $L_{2}$-fitting algorithm is simple and practical, whereas the $L_{1}$-fitting algorithm depends on using a semi-dynamic range search data structure and parametric search. For general $k$, we present two approximation schemes. Let $z_{\text {opt }}$ be the minimum fitting error for a $k$-joint polyline and let $\varepsilon$ be a positive constant. We give a polynomial-time approximation scheme (PTAS) to compute a $\lfloor(1+\varepsilon) k\rfloor$-joint fitting whose error is at most $z_{\text {opt }}$ and we describe a fully polynomial-time approximation scheme (FPTAS) to compute a $k$-joint polyline with $(1+\varepsilon) z_{\text {opt }}$ fitting error, and consequently show that the problems cannot be strongly NP-hard, although their NP-hardness remains open. BorIS SAYS: I asked before: "Can we do 1+eps approximation of BOTH?" We do not have it in the paper now, but

\footnotetext{
${ }^{1}$ We write $f(n)=\tilde{O}(g(n))$ if there exists an absolute constant $c \geq 0$ such that $f(n)=O\left(g(n) \log { }^{c} n\right)$.
} 
perhaps we should?

Intuitively, why are the problems we consider in this paper more difficult than some related questions? We have mentioned that the uniform metric fitting problem can be solved efficiently. The key point is that its corresponding decision problem to determine whether there exists a $k$ joint polyline with a uniform error less than a given value $w$ is geometrically a stabbing problem. The $k$-joint path must go through $n$ vertical line segments of length $2 w$ centered at the input points, and one can continuously move a feasible polyline so that each link becomes extremal in geometric sense, that is, goes through a pair of endpoints of vertical segments. Hence, we can design a polynomial-time algorithm to find the optimal path based on dynamic programming. The $L_{1}$ - and $L_{2}$-fitting problems seem to be more subtle: We do not have reformulations of the corresponding decision problems in terms of stabbing.

\section{Preliminaries}

A $k$-joint polyline is an alternating sequence $P=\left(e_{1}, \mathbf{v}_{1}, e_{2}, \mathbf{v}_{2}, \ldots, e_{k}, \mathbf{v}_{k}, e_{k+1}\right)$ of line segments (links) and joint vertices (joints), where $e_{s}$ and $e_{s+1}$ share the endpoint $\mathbf{v}_{s}$, for $s=1,2, \ldots, k$, and $e_{1}$ and $e_{k+1}$ are infinite rays. We denote the link $e_{s}$ on line $y=a_{s} x-b_{s}$ by $\left(a_{s}, b_{s}\right)$ if the interval of the values of $x$ corresponding to the link is understood. A joint $\mathbf{v}_{s}$ is represented by the pair $\left(u_{s}, v_{s}\right)$ of its coordinate values. Thus, the connectivity and monotonicity of the polyline can be guaranteed by requiring that $v_{s}=a_{s} u_{s}-b_{s}=a_{s+1} u_{s}-b_{s+1}$, for $s=1,2, \ldots, k+1$, and $u_{1}<\ldots<u_{k}$. BORIS SAYS: Technically, this is wrong, as the last equality only works up to $s=k$ not $s=k+1$.

We now formulate the problem of fitting a $k$-joint polyline to an $n$-point set. Given a set of points $S=\left\{p_{1}=\left(x_{1}, y_{1}\right), p_{2}=\left(x_{2}, y_{2}\right), \ldots, p_{n}=\left(x_{n}, y_{n}\right)\right\}$ with $x_{1}<x_{2}<\ldots<x_{n}$ and an integer $k$, and setting $u_{0}=-\infty$ and $u_{k+1}=\infty$ for convenience, find a polyline $P=$ $\left(\left(a_{1}, b_{1}\right),\left(u_{1}, v_{1}\right),\left(a_{2}, b_{2}\right),\left(u_{2}, v_{2}\right), \ldots,\left(u_{k}, v_{k}\right),\left(a_{k+1}, b_{k+1}\right)\right)$ minimizing one of the following three quantities for $L_{1^{-}}, L_{2^{-}}$, and uniform metric fitting, respectively:

$$
\begin{gathered}
L_{1}: \sum_{s=1}^{k+1} \sum_{u_{s-1}<x_{i} \leq u_{s}}\left|a_{s} x_{i}-b_{s}-y_{i}\right|, \\
L_{2}: \sum_{s=1}^{k+1} \sum_{u_{s-1}<x_{i} \leq u_{s}}\left(a_{s} x_{i}-b_{s}-y_{i}\right)^{2}, \\
\text { Uniform metric: } \max _{s=1, \ldots, k+1}\left\{\max _{u_{s-1} \leq x_{i} \leq u_{s}}\left|a_{s} x_{i}-b_{s}-y_{i}\right|\right\} .
\end{gathered}
$$

For $k=0$, the problems are linear regression problems. The $L_{2}$-linear regression is well known as the Gaussian least-squares method. Once we compute $\mathrm{A}_{n}=\sum_{i=1}^{n} x_{i}, \mathrm{~B}_{n}=\sum_{i=1}^{n} y_{i}$, $\mathrm{C}_{n}=\sum_{i=1}^{n} x_{i}^{2}, \mathrm{D}_{n}=\sum_{i=1}^{n} x_{i}^{2}$, and $\mathrm{E}_{n}=\sum_{i=1}^{n} x_{i} y_{i}$ in linear time, we can construct an optimal fitting line $y=a x-b$ by considering the partial derivatives of the objective function and solving a $2 \times 2$ system of linear equations. The linear regression problem with respect to the uniform error is equivalent to finding a pair of parallel lines at the minimum vertical distance that contain all the given points between them. This can be done by applying the rotating caliper method that computes antipodal pairs of points on the convex hull of the point set. For an $x$-sorted point set this can be done in $O(n)$ time [24]. The $L_{1}$-linear regression problem is more involved; however, a linear-time algorithm has been devised by Imai et al. [13] based on Megiddo's prune-and-search paradigm. 


\section{$3 \quad$ Fitting a 1-joint polyline}

We consider the problem of fitting a 1-joint polyline to a set of points. We proceed in two steps. We first assume that the joint vertex lies in a fixed interval $\left[x_{q}, x_{q+1}\right]$ and later eliminate this assumption. Let $S_{1}(q)=\left\{p_{1}, p_{2}, \ldots, p_{q}\right\}$ and $S_{2}(q)=\left\{p_{q+1}, \ldots, p_{n}\right\}$. Our objective polyline consists of two links lying on lines $\ell_{1}: y=a_{1} x-b_{1}$ and $\ell_{2}: y=a_{2} x-b_{2}$, respectively. We call a tuple $\left(a_{1}, b_{1}, a_{2}, b_{2}\right)$ feasible if the two lines $y=a_{1} x-b_{1}$ and $y=a_{2} x-b_{2}$ meet at a point whose $x$-coordinate $u=\frac{b_{1}-b_{2}}{a_{1}-a_{2}}$ lies in the interval $\left[x_{q}, x_{q+1}\right]$. Our goal here is to find a feasible tuple $\left(a_{1}, b_{1}, a_{2}, b_{2}\right)$ representing a 1 -joint polyline minimizing

$$
\begin{aligned}
& \sum_{i=1}^{q}\left|a_{1} x_{i}-b_{1}-y_{i}\right|+\sum_{i=q+1}^{n}\left|a_{2} x_{i}-b_{2}-y_{i}\right| \text { and } \\
& \sum_{i=1}^{q}\left(a_{1} x_{i}-b_{1}-y_{i}\right)^{2}+\sum_{i=q+1}^{n}\left(a_{2} x_{i}-b_{2}-y_{i}\right)^{2},
\end{aligned}
$$

for $L_{1^{-}}$and $L_{2}$-fitting, respectively. Minimizing (4) is equivalent to, provided $a_{1} \neq a_{2}$, minimizing $\sum_{i=1}^{n} w_{i}$ subject to

$$
\begin{aligned}
& -w_{i} \leq a_{1} x_{i}-b_{1}-y_{i} \leq w_{i}, \quad \text { for } i \leq q, \\
& -w_{i} \leq a_{2} x_{i}-b_{2}-y_{i} \leq w_{i}, \quad \text { for } i \geq q+1, \quad \text { and } \\
& x_{q} \leq \frac{b_{1}-b_{2}}{a_{1}-a_{2}} \leq x_{q+1},
\end{aligned}
$$

where the last line represents the feasibility condition.

Lemma 3.1. For either $L_{1}$ - or $L_{2}$-fitting criterion, the 1 -joint problem for a fixed $q$ reduces to solving two convex programming problems.

Proof. Disregarding the feasibility constraint, the problem is clearly a quadratic programming problem for the $L_{2}$ case and a linear programming problem for the $L_{1}$ case. The feasibility constraint requiring that the lines $\ell_{1}$ and $\ell_{2}$ meet in the strip between $x_{q}$ and $x_{q+1}$ can be expressed by different linear constraints depending on whether $a_{1} \leq a_{2}$. Thus, we can decompose the $\left(L_{1}\right.$ or $\left.L_{2}\right)$ problem into two subproblems. If $a_{1} \leq a_{2}$, the lines meet in the strip if and only if $\ell_{1}$ is not below $\ell_{2}$ at $x_{q}$ and is not above it at $x_{q+1}$. Thus, the additional constraint becomes

$$
x_{q}\left(a_{2}-a_{1}\right) \leq b_{2}-b_{1} \leq x_{q+1}\left(a_{2}-a_{1}\right) .
$$

In the opposite case, the additional constraint is

$$
x_{q+1}\left(a_{2}-a_{1}\right) \leq b_{2}-b_{1} \leq x_{q}\left(a_{2}-a_{1}\right) .
$$

Clearly, each subproblem is a convex programming problem, as claimed.

From the above lemma, it is clear that the optimal 1-joint polyline can be computed by using linear/quadratic programming. However, we aim to design combinatorial algorithms for these problems. Indeed, we can classify the solution into two types: (a) An inequality in (7), (8) holds with equality. (b) All of the inequalities in (7), (8) are strict. We call the solution fixed in the former case and free otherwise. From the form of the expressions in (7), (8) we deduce the following simple observation.

Lemma 3.2. If the solution is fixed, the joint is located on either of the two vertical lines $x=x_{q}$, $x=x_{q+1}$. 
If the joint is on the line $x=x_{q+1}$, we can regard it as a solution for the partition into $S_{1}(q+1)=S_{1}(q) \cup\left\{p_{q+1}\right\}$ and $S_{2}(q+1)=S_{2}(q) \backslash\left\{p_{q+1}\right\}$. Thus, for each partition, we essentially need to solve two subproblems: (1) the free problem and (2) the fixed problem where the joint is on the vertical line $x=x_{q}$. This leads to the following generic algorithm: For each partition of $S$ into two intervals $S_{1}$ and $S_{2}$, we first consider the free problem ignoring the feasibility constraint, and check whether the resulting solution is feasible, i.e., we verify that the intersection point lies in the strip between $p_{q}$ and $p_{q+1}$. If it is feasible, it is the best solution for the partition. Otherwise, we consider the fixed solution adding the constraint that the joint lie on $x=x_{q}$, and report the solution for the partition. After processing all $n-1$ possible partitions, we report the solution with the smallest error.

If it takes $O(f(n))$ time to process a subproblem for each partition, the total time complexity is $O(n f(n))$. For efficiency, we design a dynamic algorithm to process each partition so that $f(n)$ is reduced in the amortized sense.

\subsection{The $L_{2}$ 1-joint problem}

We show how to construct an optimal $L_{2}$-fitting 1 -joint polyline in linear time. We process the partitions $\left(S_{1}(q), S_{2}(q)\right)$ starting from $q=1$ to $q=n-1$, in order. We maintain the sums, variances, and covariances $\mathrm{A}_{q}=\sum_{i=1}^{q} x_{i}, \mathrm{~B}_{q}=\sum_{i=1}^{q} y_{i}, \mathrm{C}_{q}=\sum_{i=1}^{q} x_{i}^{2}, \mathrm{D}_{q}=\sum_{i=1}^{q} y_{i}^{2}$, and $\mathrm{E}_{q}=\sum_{i=1}^{q} x_{i} y_{i}$ incrementally, at constant amortized cost. They also provide us with the corresponding values for $S_{2}(q)$ if we precompute those values for $S$, i.e., $\sum_{i=q+1}^{n} x_{i}=\mathrm{A}_{n}-\mathrm{A}_{q}$ etc.

For the free case, the objective function is separable, in the sense that the optimal solution can be identified by finding $\left(a_{1}, b_{1}\right)$ minimizing $\sum_{i=1}^{q}\left(a_{1} x_{i}-b_{1}-y_{i}\right)^{2}$ and $\left(a_{2}, b_{2}\right)$ minimizing $\sum_{j=q+1}^{n}\left(a_{2} x_{j}-b_{2}-y_{j}\right)^{2}$ independently. Each can be computed in $O(1)$ time from the values of $\mathrm{A}_{q}, \ldots, \mathrm{E}_{q}$ as explained in section 2 . The feasibility check of the solution is done in $O(1)$ time by computing the intersection point of the corresponding pair of lines. It remains to solve the subproblems with the additional constraint that the joint is at $x=x_{q}$. Put

$$
\begin{aligned}
& f\left(a_{1}, b_{1}, a_{2}, b_{2}\right)=\sum_{i=1}^{q}\left(a_{1} x_{i}-b_{1}-y_{i}\right)^{2}+\sum_{j=q+1}^{n}\left(a_{2} x_{j}-b_{2}-y_{j}\right)^{2}, \\
& g\left(a_{1}, b_{1}, a_{2}, b_{2}\right)=a_{1} x_{q}-b_{1}-a_{2} x_{q}+b_{2}, \text { and } \\
& L\left(a_{1}, b_{1}, a_{2}, b_{2}\right)=f\left(a_{1}, b_{1}, a_{2}, b_{2}\right)-\lambda g\left(a_{1}, b_{1}, a_{2}, b_{2}\right),
\end{aligned}
$$

so that $f(\cdot)$ is the function to be minimized and the joint constraint can be expressed as $g(\cdot)=0$. Then, by the Kuhn-Tucker condition the optimal solution $Z_{\mathrm{opt}}=\left(a_{1}^{0}, b_{1}^{0}, a_{2}^{0}, b_{2}^{0}\right)$ describing a best $L_{2}$-fitting 1-joint polyline for a fixed value of $q$ has to satisfy

$$
\left.\frac{\partial L}{\partial a_{1}}\right|_{Z_{\mathrm{opt}}}=\left.\frac{\partial L}{\partial b_{1}}\right|_{Z_{\mathrm{opt}}}=\left.\frac{\partial L}{\partial a_{2}}\right|_{Z_{\mathrm{opt}}}=\left.\frac{\partial L}{\partial b_{2}}\right|_{Z_{\mathrm{opt}}}=0
$$

and

$$
g\left(Z_{\text {opt }}\right)=0
$$

This gives us a set of five linear equations that must be satisfied by the optimal parameter values of $a_{1}, b_{1}, a_{2}, b_{2}$ and the Lagrange multiplier $\lambda$. The coefficients can be expressed in terms of $x_{q}, \mathrm{~A}_{q}, \ldots, \mathrm{E}_{q}$, and this system can be solved in constant time for each $q$. Thus, we have the following:

Theorem 3.3. $L_{2}$-optimal 1-joint fitting can be computed in linear time. 


\subsection{The $L_{1}$ 1-joint problem}

\subsubsection{Semi-dynamic $L_{1}$ linear regression}

We start with the problem of computing the optimal linear $L_{1}$-fitting (i.e., linear regression) of the input point set, i.e., we seek the line $\ell_{\mathrm{opt}}: y=a x-b$ minimizing $\sum_{i=1}^{n}\left|a x_{i}-b-y_{i}\right|$.

BORIS SAYS: In the next two sentences the word "problem" appears 4 times. A bit too much. The difficulty with the $L_{1}$-fitting problem is that, written in linear programming terms (as in (6)), it has $n+2$ variables, in contrast to the least-squares case where the problem is directly solved as a bivariate problem. Nonetheless, the problem can be solved by a brute-force combinatorial algorithm in $O\left(n^{3}\right)$ time, since there are $O\left(n^{2}\right)$ possible linear dissections of the point set which can be enumerated in $\Theta\left(n^{2}\right)$ worst-case time by constructing the dual arrangement, and one can compute the optimal line in linear time once the dissection by the line is given (this algorithm can be easily sped up to constant or near-constant amortized time per dissection). Moreover, by Lemma 3.4, the optimal line bisects $S$ into two equal-size subsets; in other words, the line is a halving line. Using this fact, Imai et al. [13] devised an optimal linear-time algorithm for computing $\ell_{\text {opt }}$ based on the multidimensional prune-and-search paradigm. In order to design an efficient algorithm for the 1-joint fitting problem, we consider a semi-dynamic version of the $L_{1}$ linear regression for a point set $P$ with low amortized time complexity, where we dynamically maintain $P$ with insertions and deletions under an assumption that $P$ is always a subset of a fixed universe $S$ of size $n$ that is given from the outset. (In fact, for our application, it is sufficient to be able to start with $P=\emptyset$ and handle only insertions, and to start with $P=S$ and handle only deletions. Moreover, the order of insertions and deletions is known in advance. The data structure we describe below is more general.)

Consider the dual space, with $p_{i}=\left(x_{i}, y_{i}\right)$ transformed to the dual line $Y=f_{i}(X)$ where $f_{i}(X)=x_{i} X-y_{i}$. The line $y=a x-b$ is transformed to the point $(a, b)$ in the dual space. The $k$ th level of the arrangement $\mathcal{A}=\mathcal{A}\left(S^{*}\right)$ of the set $S^{*}$ of dual lines is the trajectory of the $k$ th largest value among $f_{i}(X) .^{2}$ We call the $\lceil n / 2\rceil$ th level the median level.

Lemma 3.4 (Imai et al. [13]). If the optimal $L_{1}$-fitting line is given by $y=a_{\mathrm{opt}} x-b_{\mathrm{opt}}$, its dual point $\left(a_{\mathrm{opt}}, b_{\mathrm{opt}}\right)$ is on the median level if $n$ is odd, and between the $\frac{n}{2}$ th level and the $\left(\frac{n}{2}+1\right)$ th

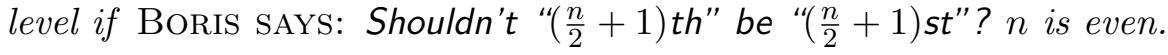

In case the optimal slope is not unique, let $a_{\text {opt }}$ denote the smallest such slope. Now, given $X$-value $t$, consider the point $\left(t, f_{i}(t)\right)$ for each $i=1,2, \ldots, n$, and let $F(t)$ be the sum of the $\lfloor n / 2\rfloor$ largest values in $\left\{f_{i}(t): i=1,2, \ldots, n\right\}$ and $G(t)$ be the sum of the $\lfloor n / 2\rfloor$ smallest values in the same set. Put $H(t)=F(t)-G(t) . H(t)$ gives the $L_{1}$ fitting error of the dual line of any point $(t, y)$ on the median level (or between the two median levels if $n$ is even). Thus, by Lemma 3.4, $H(t)$ is minimized at $t=a_{\mathrm{opt}}$.

Lemma 3.5. $F(t)$ is a convex function, while $G(t)$ is concave. As a consequence, $H(t)$ is also convex. At $t=a_{\mathrm{opt}}$ the slope of $H(t)$ changes from negative to non-negative.

Proof. The convexity follows directly from the fact that, in any line arrangement, the portions of the lines lying on or below (resp. on or above) any fixed level $k$ can be decomposed into $k$ non-overlapping concave (resp. convex) chains; see, for example, [3].

Suppose a fixed universe $S^{*}$ of lines is given. We need a data structure that maintains a subset $P^{*} \subseteq S^{*}$ and supports the following operations on $P^{*}$ :

Median-location query For a query value $t$, return the point on the $\lfloor n / 2\rfloor$ th highest line at $X=t$.

\footnotetext{
${ }^{2}$ We use an asterisk to denote geometric dual of a point, line, or a set of lines/points.
} 
Slope-sum query For a query point $p=(t, y)$, return the sum of the slopes of lines below $p$ at $X=t$.

Height-sum query For a query value $p=(t, y)$, return the sum of the $Y$-coordinates of the lines below $p$ at $X=t$. The height-sum query is reduced to a slope-sum query plus a constantterm-sum query that reports the sum of the constant terms of the equations representing lines.

Update A line in $S^{*}$ is added to or removed from $P^{*}$.

Suppose a data structure supporting such queries on a set $P^{*} \subseteq S^{*}$ of lines in $O(\tau(n))$ time is available, where $n=\left|S^{*}\right|$. Then we can query the slopes of $F$ and $G$ at $t$, and hence compute the slope of $H$ at $t$ in $O(\tau(n))$ time.

TAKESHI SAYS: I add some explanation for the case where we encounter a break point. We indeed need not consider slope if we apply perturbation, but I do not change in that way. BORIS SAYS: Slightly rephrased

It may happen that the piecewise linear function $H$ has a break point at $t$. In order to handle this case, we apply symbolic perturbation and examine the slopes of $H$ at $t+\epsilon$ and $t-\epsilon$ for an infinitesimally small $\epsilon>0$ to determine whether $H$ increases to the right of $t$, to the left of $t$, or in both directions. This perturbation is only activated when a linear decision required in queries needs a tie-break. Because of convexity of $H$, we have the following:

Lemma 3.6. Given $t$, we can decide whether $t<a_{\mathrm{opt}}, t>a_{\mathrm{opt}}$, or $t=a_{\mathrm{opt}}$ in $O(\tau(n))$ time.

Thus, we can perform binary search to find $a_{\text {opt }}$. We show below how to make this search strongly polynomial. Once we know $a_{\text {opt }}$, we determine $b_{\text {opt }}$ by the median-location query at $t=a_{\mathrm{opt}}$.

\subsubsection{Semi-dynamic data structure for the queries}

We show how to realize semi-dynamic median-location- and sum-queries. As a preliminary step, we describe a semi-dynamic data structure for vertical ray queries, i.e., queries of the form: Given a vertical upward ray starting at $(t, z)$ determine the number of lines in $P^{*}$ intersected by the ray, the sum of their slopes, and the sum of their constant terms. A dual line $Y=x_{i} X-y_{i}$ is above $(t, z)$ iff the primal point $\left(x_{i}, y_{i}\right)$ is above the line $y=t x-z$. Thus our queries are reduced to half-space queries in the primal plane. We use the partition-tree data structure of Matoušek $[4,17,19]$. It supports half-space queries on sets with $n$ points in time $O(\sqrt{n})$, linear space, and preprocessing time $O(n \log n)$.

We build a partition tree $\mathcal{T}(S)$ on the set $S$ of points dual to the lines in $S^{*}$ (in fact, these are the points to which a line is being fitted). A standard construction proceeds as follows: With each node $v$ of the partition tree we associate a point set $S(v) \subseteq S$ and a triangle $\Delta(v) \supset S(v)$, where $S(v) \subset S($ parent $(v))$ at any node $v$ other than the root and $S(v)=S$ at the root. In addition we also store at $v$ the size $|S(v)|$ of $S(v)$ and the sums $\xi(S(v))=\sum_{p_{i} \in S(v)} x_{i}$ and $\chi(S(v))=\sum_{p_{i} \in S(v)} y_{i}$ of the slopes and constant terms of the corresponding dual lines. Since the point sets $S(v)$, over all children $v$ of a node $w$ in the tree, by definition of a partition tree, partition the set $S(w)$, and $|S(v)|$ is at most a fraction of $|S(w)|$, this tree has linear size and logarithmic depth. For our purposes, we modify the partition tree to obtain a new tree $\mathcal{T}(S, P)$ where the same $\Delta(v)$ as in $\mathcal{T}(S)$ is associated with every node $v$, but $v$ stores $P(v)=S(v) \cap P$, $\xi(P(v))$ and $\chi(P(v))$ instead of the corresponding values for $S(v)$. This data structure enables us to execute the half-plane range query in $P$, and thus the vertical ray query in $P^{*}$.

Our data structure is semi-dynamic. When $P$ changes, with a point $p$ being added or removed, what we need to update is just values $|P(v)|, \xi(P(v))$, and $\chi(P(v))$ for each node $v$ where $p$ is 
relevant. Since the sets $S(v)$ for all nodes $v$ at a fixed level of the partition tree form a partition of $S$, only one node must be updated at each level; to facilitate the update one might associate with each point $p \in S$ a list of length $O(\log n)$ containing the nodes $v$ of the tree with $p \in S(v)$. BorIS SAYS: Doesn't this decision increase the space to $\Theta(n \log n)$. Thus, the update can be performed in $O(\log n)$ time. This ends the description of the semi-dynamic vertical ray query data structure. Our sum-queries can be done by using the vertical ray query.

We next turn to the median-location query data structure. For a given $t$, let $m(t)=(t, y(t))$ be the intersection of the vertical line $X=t$ and the median level of the dual arrangement $\mathcal{A}\left(S^{*}\right)$. We can use the vertical query data structure to compare any given $\eta$ with $y(t)$. We perform a vertical ray query to find the number of lines above $(t, \eta)$. If it is less than $\lfloor n / 2\rfloor, y(t)<\eta$; otherwise $y(t) \geq \eta$. This suggests computing $y(t)$ by some kind of binary search. If we had the sorted list of intersections between the vertical line $X=t$ and the lines in $S^{*}$ available, we could perform a binary search on $L$ by using $O(\log n)$ ray queries. However, it takes $O(n \log n)$ time to compute the list, which is too expensive since we aim for a sublinear query time. Instead, we construct a data structure which can simulate the binary search without explicitly computing the sorted list.

Lemma 3.7. We can construct a randomized data structure in time $O(n \log n)$ such that, given $t$, we can compute $y(t)$ in $O(\sqrt{n} \log n)$ time. The query time bound holds for every vertical line $X=t$ with high probability.

Proof. TAKeshi SAYs: I add some remarks on dynamization. Boris SAYs: Rephrased. Recall that we start with an underlying fixed set $S$ of points and aim to build a semi-dynamic data structure for answering queries on a subset $P^{*}$ of the set $S^{*}$ of dual lines.

We fix a small constant $\varepsilon>0$, and randomly select $c n^{1-\varepsilon}$ lines from $\Psi_{0}=S^{*}$, to have a set $\Psi_{1}$ of lines, where $c$ is a suitable constant. From the results of Clarkson and Shor [9], if the constant $c$ is sufficiently large, with high probability every vertical segment intersecting no line of $\Psi_{1}$ intersects at most $n^{\varepsilon} \log n$ lines of $S^{*}$. In other words, $\Psi_{1}$ is the dual of an $\left(n^{\varepsilon-1} \log n\right)$-net of $S$. Similarly, we construct $\Psi_{i+1}$ from $\Psi_{i}$ such that $\Psi_{i+1}^{*}$ is a $\min \left\{\frac{n^{\varepsilon} \log n}{\left|\Psi_{i}\right|}, 1\right\}$-net of $\Psi_{i}^{*}$. Thus, we have a filtration $\Psi_{0} \supset \Psi_{1} \supset \ldots \supset \Psi_{k}$, and $\left|\Psi_{k}\right| \leq n^{\varepsilon}$. The number $k$ of layers is a function of $\varepsilon$ and $c$ only, so the construction takes $O(n)$ time.

Additionally, we construct a dual range-searching data structure for $\Psi_{i}$ such that for a query vertical interval $I$ we can report all lines in $\Psi_{i}$ meeting $I$ in $O(\sqrt{n}+K)$ time, where $K$ is the number of reported lines. In primal space a vertical interval corresponds to a strip bounded by two parallel lines and hence we may use partition trees as described above to implement reporting queries. The preprocessing time is $O(n \log n)$.

Now, our algorithm for finding $y(t)$ is as follows: Given $t$, we first compute all the intersections between $X=t$ and the lines of $\Psi_{k}$, sort them to have a list $y^{(1)}, y^{(2)}, \ldots y^{(m)}$ of $y$-values where $m \leq n^{\epsilon}$. By using the semi-dynamic vertical ray-query data structure for $P^{*}$, we can decide whether $y(t)>y^{(i)}$ or BORIS SAYs: I changed $y(t) \leq y^{(i+1)}$ to $y(t) \leq y^{i}$, is this ok? $y(t) \leq y^{i}$ in $O(\sqrt{n})$ time. Accordingly, we can perform binary search to find the unique interval $I_{k}=$ $\left(y^{(i)}, y^{(i+1)}\right]$ containing $y(t)$ in $O(\sqrt{n} \log n)$ time.

The interior of the vertical interval $I_{k}$ containing $y(t)$ is crossed by no line of $\Psi_{k}$. By using the dual range-searching data structure, we extract, in time $O(\sqrt{n}+K)$, the set of $K$ lines in $\Psi_{k-1}$ intersecting $I_{k} ; K=O\left(n^{\varepsilon} \log n\right)$ with high probability. BorIS SAYs: Added... Starting with $I_{k}$ and the extracted set of $K$ lines, we compute $I_{k-1} \subseteq I_{k}$ not intersected by any line of $\Psi_{k-1}$. Proceeding recursively, we eventually obtain $y(t)$, since at the last level of the filtration we arrive at an interval $I_{0}$ containing $y(t)$, with no line of $\Psi_{0}=S^{*}$ crossing its interior. The total time is $O\left(n^{\varepsilon} \log ^{2} n+\sqrt{n} \log n\right)=O(\sqrt{n} \log n)$. 
We have described an $O(\sqrt{n} \log n)$ time realization of the semi-dynamic query data structure, i.e., $\tau(n)=O(\sqrt{n} \log n)$ in this data structure.

\subsubsection{Determining $a_{\mathrm{opt}}$}

We finally come to the strongly polynomial method for determining $a_{\text {opt }}$ via parametric search [25]. We remark that we do not employ parametric search to compute $y(t)$ for a fixed $t$, since it is not always possible to use parametric search in a nested fashion, and there are technical difficulties in applying multi-dimensional parametric search paradigm $[20,26]$ to our problem.

Parametric search identifies a real number $a_{\text {opt }}$. The search has two ingredients:

- A decision procedure $D(t)$ of one real parameter. The procedure tests whether $t<a_{\mathrm{opt}}$ in time $O\left(T_{D}\right)$.

- A master program $M(t)$ which takes as input a real parameter $t \in(-\infty, \infty) . M(t)$ is a parallel program which takes time $T_{M}$ with $p$ processors for any fixed value of $t$. We assume that $M(t)$ proceeds in parallel rounds, as follows: Each processor performs some calculation and then makes a decision that depends on whether or not the input value $t$ is larger than a just computed threshold value which does not depend on $t$ (more generally, there may be a constant number of such threshold values per processor per round and the decision depends on where $t$ lies among them). Once the decisions are made by all processors, the next parallel round begins. Thus, in each round of the parallel program, we have at most $p$ threshold values that subdivide $(-\infty, \infty)$ into at most $p+1$ subintervals, and all decisions in the round are determined by the subinterval containing $t$. We assume subintervals to be open on the left and closed on the right and we require that $a_{\mathrm{opt}}$ is generated as a threshold value when $M(t)$ is run with $t=a_{\mathrm{opt}}$. In this case, the final interval computed by the procedure described below has $a_{\text {opt }}$ as its right boundary.

Parametric search simulates the execution of $M(t)$ for $t=a_{\text {opt }}$. We maintain an interval $I$ (open on the left and closed on the right) containing $a_{\text {opt }}$ and a set $Q$ of real numbers. Initially, $I=(-\infty, \infty)$ and $Q=\emptyset$. We simulate $M(t)$, one round at a time. In each round, we generate the threshold value of each processor and, if it is contained in $I$, insert it into $Q$. Then, we compute the median $m$ of $Q$ and compare $m$ with $a_{\text {opt }}$ by invoking $D(m)$. If $m \geq a_{\text {opt }}$, we replace $I$ by $I \cap(-\infty, m]$, otherwise by $I \cap(m, \infty)$. The elements of $Q$ not contained in the updated $I$ are removed from $Q$. We iterate this process until $I$ has no element of $Q$ in its interior. Then, we can determine all decisions in the current round and proceed to the next round. Thus, we complete the simulation in $O\left(p T_{M}+T_{M} T_{D} \log p\right)$ time.

The decision procedure: In our case $a_{\text {opt }}$ is the slope of the optimal $L_{1}$-line. The decision procedure proceeds as follows. Let $t$ be the input. BorIS SAYS: Slight notational confusion: Earlier we used $I_{k}, I_{k-1}$ to denote the vertical interval we keep narrowing down (and its new shorter version). Here we call them $s, s^{\prime}$ and use $I$ to denote the interval of values for $a_{\mathrm{opt}}$. What do we do? If it causes not too much trouble, I suggest we leave $I$ alone (no subscripts!) and replace $s$ by $I_{k}$ and $s^{\prime}$ by $I_{k-1}$ or would this be too hard to follow?

1. Determine the $y$-coordinate $y(t)$ of the median level of the dual arrangement at $t$ using the filtration $\left(\Psi_{i}\right)$ of the dual arrangement. At level $i$ of the filtration, we have a vertical segment $s$ delimited by two lines of $\Psi_{i}$ and not properly intersected by any line of $\Psi_{i}$. BORIS SAYS: Replaced $\Psi_{i+1}$ by $\Psi_{i-1}$ throughout, as they are numbered from the largest to the smallest. 
(a) First determine the lines of $\Psi_{i-1}$ intersecting $s$. This is a range query, where the range is the strip defined by the two primal parallel lines dual to the endpoints of $s$. The running time is $O(\sqrt{n}+K)$ where $K=\tilde{O}\left(n^{\varepsilon}\right)$ is the number of lines intersected. What kinds of comparisons are we making here? We have a line $t x+b$, for given $b$, and we check whether this line intersects a triangle. This can be rewritten as a comparison of $t$ with a real number.

(b) Once we have determined the intersected lines, we sort the intersections and perform binary search on them. For each point, we need to count the number of lines above it. This is again a range query. So with $\log n$ "number of lines above a point" queries, we have reduced $s$ to $s^{\prime}$ delimited by two adjacent lines of $\Psi_{i-1}$.

The comparison of two lines at the vertical line $t$, or of $a t+b$ with $a^{\prime} t+b^{\prime}$, is equivalent to comparing $t$ with a real number.

2. Once we have found $y(t)$, we use a single slope query and a single constant term query to determine the slope of $H(t)$ at $t$. The sign of this slope determines whether $t<a_{\mathrm{opt}}$.

The Master Program: The master program is simply the parallel version of the decision procedure. TAKESHI SAYS: I gave minor change. BORIS SAYS: slightly reworded It uses $O(\tau(n))$ processors, where $\tau(n)$ is the sequential query time. We first present the version with $\tau(n)=$ $O(\sqrt{n} \log n)$, and refine our description below. The range queries are easily parallelized because they amount to walking down at most $\log n$ paths in the partition tree. Thus the parallel time of a range query is $O(\log n)$. Step 1(b) amounts to a parallel sort (time $O(\log n)$ ) followed by a binary search $($ time $O(\log n))$. Each step of the binary search requires a range query. Step 2 is also a single range query. Thus the parallel time is $O\left(\log ^{2} n\right)$ with $O(\tau(n))$ processors. When executed with $t=a_{\mathrm{opt}}$, the master program generates $a_{\mathrm{opt}}$ as a threshold value since two dual lines intersect at $a_{\mathrm{opt}}$.

Some Intuition: It is instructive to see what the parametric search does geometrically. In each $\Psi_{i}$ it determines a trapezoid $T$ with two vertical walls (sides) and two non-vertical edges (top and bottom). The non-vertical edges lie on adjacent lines in $\Psi_{i}$. The median level of the full arrangement intersects the sides and avoids the top and bottom. In addition, $a_{\mathrm{opt}}$ lies in the $x$-span $I$ of $T$. The interval $I$ is the interval maintained in the parametric search.

In step 1(a), we narrow $T$ by moving in its vertical walls, which ensures that no lines of $\Psi_{i-1}$ enters the reduced trapezoid through its top and bottom. In other words, all vertical segments connecting the top to the bottom of the trapezoid intersect the same set of lines of $\Psi_{i-1}$.

Next we come to sorting the intersections (step 1(b)) with the generic vertical segment spanned by $T$. Step 1(b) will further move in the vertical walls until the trapezoid contains no intersections between lines in $\Psi_{i-1}$.

At this point the lines in $\Psi_{i-1}$ are sorted and we can perform binary search on them. Each search step will move one of the non-vertical walls. Also in each search step we do a range search and this may further move in the vertical walls (since the median level must stay within the shrinking trapezoid).

This completes our description of the parametric-search-based procedure for determining $a_{\mathrm{opt}}$.

\subsubsection{Time-space tradeoff}

To speed up the query time $\tau(n)$ and thus the overall algorithm, we generalize the data structure to allow it to use super-linear storage based on Matoušek's construction [18]. If we can use $O(m)$ space for $n<m<n^{2}$, we first select $r=O(m / n)$ points from $S$ and construct a dual cutting, i.e., a decomposition of the dual plane into cells, such that each cell $C$ is intersected by at most 
$n / r$ lines dual to points of $S$; the number of cells required is $O\left(r^{2}\right)$ and the computation time is $O(n r)$.

Let $S(C)$ be the set of lines intersecting $C$. We construct a point-location data structure on the cutting. For each cell $C$, we store the cumulative statistics (the sum of slopes etc.) for the set of lines passing below $C$, and construct the partition tree for $S(C)$. The query time of each tree is $\tilde{O}(\sqrt{n / r})$. When $P$ changes, we need to update the data stored in each of the $O\left(r^{2}\right)$ cells of the cutting, and also the $O(r)$ partition trees corresponding to sets containing the updated point. Thus, update time is $O\left(r^{2}+r \log n\right)$.

Update time can be sped up by not storing the statistics for each cell explicitly, but rather retrieving them when needed at a cost of $O(\log r)$. This reduces the time needed for an update to $O(r \log r)$ as shown below. Thus, if we set $r=n^{1 / 3}$, the update time and query time is $\tau(n)=\tilde{O}\left(n^{1 / 3}\right)$. The space and preprocessing time is $\tilde{O}\left(n^{4 / 3}\right)$. The parallel time complexity is not affected by the space-time trade-off.

Now we explain how to reduce the update time to $O(r \log r)$. The issue is that each of $O\left(r^{2}\right)$ cells in the cutting has an attached data structure (partition tree) and some additional constantsize cumulative statistics (total number of lines passing below/above the cell and sums of their slopes and constant terms). Let $\ell$ be the line inserted into or deleted from $P$ in the current update. The partition trees are easy to update when a line is inserted/removed and there are only $O(r)$ of them to modify; indeed, only the partition trees stored at cells intersected by $\ell$ are affected, and there are $O(r)$ such cells because of the zone theorem [7] for arrangements.

On the other hand, the line $\ell$ contributes to the global statistics of $O\left(r^{2}\right)$ cells; thus we need $\Theta\left(r^{2}\right)$ time to update them directly. Instead, we store the information implicitly, so that it can be computed as needed in time $O(\log r)$.

Consider a possibly non-simple spanning path $\sigma$ in the cell adjacency graph of the cutting obtained, for example, by tracing along a spanning tree of the graph. The length of the path is $O\left(r^{2}\right)$. Number the cells in the order of their occurrence along $\sigma$; note that a cell might appear on the path more than once - only the first occurrence contains real data and subsequent occurrence are treated as dummy.

Build a balanced binary tree $B$ on top of $\sigma$. Each internal node $v$ corresponds to a collection of consecutive nodes on $\sigma$. Let the above count of $v$ be the number of lines in $P$ which both (1) pass above all the cells corresponding to the collection, and (2) do not pass above all the cells corresponding to the collection for the parent node of $v$. We call this number the above count of $v$. Symmetrically, we define the below count. The tree $B$ is static, but each node $v$ stores with it the above and below counts that are maintained dynamically.

Initialize all the counts to 0 when $P=\emptyset$. Now consider adding a line $\ell$ to $P$ (deletion is handled symmetrically). Line $\ell$ intersects $O(r)$ cells of the cutting. We can afford to compute which cells these are, explicitly (and we have to do it, to update the partition tree information). This gives $m=O(r)$ cell numbers. Sort them to obtain $c_{0}=-\infty, c_{1}, c_{2}, \ldots, c_{m}, c_{m+1}=+\infty$. Each interval $\left(c_{i}, c_{i+1}\right)$, if non-empty (i.e. if $\left.c_{i+1}>c_{i}+1\right)$, corresponds to a connected subpath of $\sigma$ lying completely to one side of $\ell$. We can test in constant time on which side it lies by checking one of its cells. Now decompose this interval into a logarithmic number of canonical ones (corresponding to nodes of $B$ ) and increment above/below counts at the $O(\log r)$ nodes. Repeat for each of the $O(r)$ subpaths. The time for an update of global counts is thus reduced to $O(r \log r)$, as claimed.

TAKESHI SAYS: Added some details on paralle algorithm. BorIS SAYS: I am confused: Why do we need any of this at all? Don't we need SOME master algorithm that makes a decision at the value we are looking for, and it need not be related to the decision algorithm? Why can't we just use the old master algorithm? Or is the number of processors too large? Perhaps we should explain something? Since we apply parametric searching later, we need to have a parallel algorithm for the range query with the time-space tradeoff. Note that we do not need parallel algorithms for construction 
and update of the data structure. For simplicity, we consider the counting halfplane range query. We first find in the arrangement of $r$ lines the cell $C$ containing the dual point of the line defining the halfplane. This is point location, and can be done in $O(\log n)$ time by a single processor. The retrieval of the cumulative statistics of $C$ can be also done in $O(\log n)$ time by a single processor. Now, we do range searching in $S(C)$ in time $O(\log n)$ by using $O(\tau(n))=\tilde{O}(\sqrt{n / r})$ processors as discussed in Section 3.2.3. Thus, the parallel range query is done in $O(\log n)$ time by using $O(\tau(n))$ processors. Naturally, median-location, slope-sum, and height-sum queries can be done in polylogarithmic time (indeed, $O\left(\log ^{2} n\right)$ time) by using $O(\tau(n))$ processors.

\subsubsection{Algorithm for $L_{1}$ 1-joint fitting}

TAKESHI SAYS: This subsubsecion has been considerably changed.

Finally, we describe the algorithm to find the $L_{1}$-optimal 1-joint polyline fitting a set $S$ of $n$ points in the plane. Recall that there are two different types of solutions:

Type 1 There is an index $q$ such that the 1 -joint polyline consists of the optimal $L_{1}$-fitting line of $S_{1}(q)=\left\{p_{1}, p_{2}, \ldots, p_{q}\right\}$ and that of $S_{2}(q)=\left\{p_{q+1}, p_{q+2}, \ldots, p_{n}\right\}$.

Type 2 There is an index $q$ such that the joint lies on the vertical line $x=x_{q}$.

If the optimal solution is of type 1, we compute an optimal $L_{1}$-fitting line for $S_{1}(q)$ and $S_{2}(q)$ separately, for every $q=1,2, \ldots, n$, by using the semi-dynamic algorithm with $S$ as the universe.

TAKESHI SAYS: Minor change is given. BorIS SAYs: ok Thus, the time complexity is $\tilde{O}(n \tau(n))$ for examining all $q=1,2, \ldots, n$. In particular, if we use quasi-linear space $\tilde{O}(n)$, the time complexity is $\tilde{O}\left(n^{1.5}\right)$, and if we use $O\left(n^{4 / 3}\right)$ space, the time complexity is $\tilde{O}\left(n^{4 / 3}\right)$.

TAKESHI SAYS: I gave major revision below. Otherwise, the optimal solution is of type 2 . If the index $q$ is given, our objective function is

$$
\left.f\left(a, a^{\prime}, z\right)=\sum_{i=1}^{q}\left|a\left(x_{i}-x_{q}\right)-y_{i}+z\right|+\sum_{i=q+1}^{n} \mid a^{\prime}\left(x_{i}-x_{q}\right)-y_{i}+z\right) \mid .
$$

The variable $z$ corresponds to the $y$-coordinate value of the joint, and $a$ and $a^{\prime}$ are slopes of two halflines to the left and right of the joint, respectively. BorIs SAYs: Rephrased. As a function in three variables $a, a^{\prime}, z$, the function $f$ is convex as a sum of convex terms. We want to find the triple $\left(a, a^{\prime}, z\right)$ minimizing $f$.

BORIS SAYS: (1) I removed Lemma 3.8 proving convexity of $f_{\text {opt }}$. (2) why do we need the next sentence? The restriction of $f$ obtained by fixing the variable $z$ is also a convex function in $a$ and $a^{\prime}$. Put

$$
f_{\text {opt }}(z)=\max _{a, a^{\prime}} f\left(a, a^{\prime}, z\right) .
$$

It is convex, as the maximum of a set of convex functions.

Now, suppose $\left(x_{q}, \eta_{\text {opt }}\right)$ is the location of the optimal joint. For any value $\eta$ of $z$, if we can compute $\operatorname{fopt}(\eta)$, fopt $(\eta+\epsilon)$, fopt $(\eta-\epsilon)$ for an $\epsilon>0$, we can decide whether $\eta>\eta_{\text {opt }}$ or $\eta<\eta_{\text {opt }}$, or $\eta-\epsilon \leq \eta_{\text {opt }} \leq \eta+\epsilon$. Indeed, the first case and the second case are when $\operatorname{fopt}(\eta-\epsilon)<f \operatorname{fopt}(\eta)$ and $\operatorname{fopt}(\eta+\epsilon)<\operatorname{fopt}(\eta)$, respectively, and otherwise the third case occurs. This enables us to apply binary searching and parametric searching for computing $\eta_{o p t}$.

For each $q$, we guess the $y$-coordinate value $\eta$ of the joint vertex $\left(x_{q}, \eta\right)$ and compute $f o p t(\eta)$. For the purpose, we compute the best line, in the sense of $L_{1}$ fitting, approximating each of $S_{1}(q)$ and $S_{2}(q)$ going through the (for now, fixed) joint by using almost the same strategy as in section 3.2.1.

We focus on the best line approximating $S_{1}(q)$. It suffices to determine the slope of this line. In the dual space, we just need to compute a point $p=(a(p), b(p))$ on the line $Y=x_{q} X-\eta$ such 
that $\sum_{i=1}^{q}\left|a(p) x_{i}-b(p)-y_{i}\right|$ is minimized. We observe that the above expression is convex as a function of $a$, and hence $\theta(p)=\theta^{+}(p)-\theta^{-}(p)$ is monotone and changes the sign at $p$, where $\theta^{+}(p)$ $\left(\theta^{-}(p)\right)$ is the sum of slopes of lines above $p$ (resp. below $p$ ). Thus, we can apply binary search by using slope-sum query, and this binary search can be performed in $O(\log n)$ steps by using the filtration as described in Lemma 3.7. Thus, we can compute fopt $(\eta)$ in $O\left(\tau(n) \log ^{2} n\right)$ time by using our semi-dynamic query structures, and in polylogarithmic time using $O(\tau(n))$ processors in parallel.

Now we have $f o p t(\eta)$ in hand for any given $\eta$, and we can apply binary search for computing the optimal value $\eta_{\text {opt }}$. In order to construct a strongly polynomial algorithm, we apply parametric search. Given $\eta$, our algorithm runs in polylogarithmic time using $O(\tau(n))$ processors. Thus, the parametric search paradigm [25] is applicable here. Therefore, for a fixed $q$, the second case of the problem can be handled in $\tilde{O}(\tau(n))$ time.

Since there are $n$ candidates of $q$, the overall time complexity for examining for all $q$ is $\tilde{O}(\tau(n))$. By considering the space-time tradeoff on $\tau(n)$, we have the following:

Theorem 3.8. The optimal $L_{1}$-fitting 1-joint polyline is computed in $\tilde{O}\left(n^{1.5}\right)$ randomized time using quasi-linear space, and $\tilde{O}\left(n^{4 / 3}\right)$ randomized time using $O\left(n^{4 / 3}\right)$ space.

\section{$4 \quad$ Fitting a $k$-joint polyline}

The $k$-joint fitting problem is polynomial-time solvable for fixed $k$. We describe the algorithm in a non-deterministic fashion. We guess the partition of $x_{1}, \ldots, x_{n}$ into $k$ intervals each of which corresponds to a line segment of the polyline. Also, we guess whether each joint is free or fixed. We decompose the problem at the free joints and obtain a set of subproblems. In each subproblem, we add the linear constraints corresponding to the fixed condition (i.e., each joint is located on a guessed vertical line). Thus, each subproblem is a convex programming problem: a linear program for $L_{1}$, and a quadratic program for $L_{2}$. We solve each subproblem separately to obtain the solution of the whole problem. Note that this strategy works because of the convexity of each subproblem. There are $O\left((3 n)^{k}\right)$ different choices of the guesses, thus we can be replace guessing by a brute-force search to have a polynomial-time deterministic algorithm if $k$ is a constant.

For a general $k$, we do not know whether the problem is in the class $P$ or not. Thus, we would like to consider approximation algorithms. One possible approach is to relax the requirement that number of joints be exactly $k$. We can design a PTAS for it.

Theorem 4.1. Let $z_{\mathrm{opt}}$ be the optimal $L_{1}$ (or $\left.L_{2}\right)$ error of a $k$-joint fitting. Then, for any constant $\varepsilon>0$, we can compute $a\lfloor(1+\varepsilon) k\rfloor$-joint fitting whose error is at most $z_{\mathrm{opt}}$ in polynomial time.

Proof. We ignore continuity and approximate the points by using a piecewise-linear (not necessarily continuous) function with $k$ linear pieces. This can be done by preparing the optimal linear regression for each subinterval of consecutive points of $S$, and then applying dynamic programming. We can restore continuity by inserting at most $k$ steep (nearly vertical) line segments. The resulting polyline has at most $2 k$ joints and error at most $z_{\text {opt }}$. We can improve $2 k$ to $\left\lfloor\frac{3 k}{2}\right\rfloor$ by applying the 1-joint algorithm instead of linear regression algorithm, and further improve it to $\lfloor(1+\varepsilon) k\rfloor$ by using the $r$-joint algorithm mentioned above for $r=\left\lceil\varepsilon^{-1}\right\rceil$.

Another approach is to keep the number of joints at $k$ and approximate the fitting error. We give a FPTAS for it. We only discuss the $L_{1}$ case, since the $L_{2}$ case is analogous. Let $z_{\text {opt }}$ be the optimal $L_{1}$-error, and we aim to find a $k$-joint polyline whose error is at most $(1+\varepsilon) z_{\mathrm{opt}}$. We remark that if $z_{\mathrm{opt}}=0$, our solution is exactly the same as the solution for the uniform metric fitting problem, and thus we may assume $z_{\mathrm{opt}}>0$. Recall that the uniform metric fitting problem can be solved in $O(n \log n)$ time [10]. The following is a trivial but crucial observation: 
Lemma 4.2. Let $z_{\infty}$ be the optimal error for the uniform metric $k$-joint fitting problem. Then, $z_{\infty} \leq z_{\mathrm{opt}} \leq n z_{\infty}$.

Proof. The sum of the errors in the uniform-metric-optimal polyline is at most $n z_{\infty}$. Hence $n z_{\infty} \geq z_{\text {opt }}$. On the other hand, every $k$-joint polyline has a data point in $S$ such that the vertical distance to the polyline is at least $z_{\infty}$, so $z_{\mathrm{opt}} \geq z_{\infty}$.

Our strategy is as follows: We call the $n$ vertical lines through our input points the column lines. We give a set of portal points on each column line, and call a $k$-joint polyline a tame polyline if each of its links satisfies the condition that the line containing the link goes through a pair of portal points.

On each column line, the distance between its data point and the intersection point with the optimal polyline is at most $z_{\mathrm{opt}}$, thus at most $n z_{\infty}$. Thus, on the $i$ th column line, we place the portals in the vertical range $\left[y_{i}-n z_{\infty}, y_{i}+n z_{\infty}\right]$. The portal points are placed symmetrically above and below $y_{i}$. The $j$ th portal above $y_{i}$ is located at the $y$-value $y_{i}+\left(1+\frac{\varepsilon}{2}\right)^{j-1} \delta$, where $\delta=\frac{z_{\infty} \varepsilon}{2 n}$ and $j=1,2, \ldots, M$. We choose the largest $M$ satisfying $\left(1+\frac{\varepsilon}{2}\right)^{M} \delta \leq n z_{\infty}$, and hence $M=O\left(\varepsilon^{-1} \log \left(n+\varepsilon^{-1}\right)\right)$. We also put portals at heights $y_{i}$ and $y_{i} \pm n z_{\infty}$. In this way the number of portals in any column is at most $2 M+3$.

We call a closed interval between adjacent portals in a column a prime interval.

Lemma 4.3. There exists a tame polyline whose $L_{1}$ error is at most $(1+\varepsilon) z_{\mathrm{opt}}$.

Proof. We start from the optimal polyline $\ell_{\mathrm{opt}}$, and deform it to obtain a tame polyline. We proceed sequentially, left to right. Consider the line containing the leftmost link of $\ell_{\text {opt }}$. We continuously move the line to a tame line without crossing any portal point during the movement; if the line started off passing through a portal point, we rotate it around it; if the line started off passing through two, it is already tame. The right joint of the current link is accordingly moved to the intersection of the new line and the line containing the right neighbor link. It may happen that during this transformation a joint crosses a column line. However, the intersection points of the original and the deformed polylines with a column line are located in a common prime interval. We repeat this operation, proceeding from left to right, to obtain a tame polyline.

Now, consider the change of vertical distances between a point $p_{i}$ and the two polylines. The polylines go through the same prime interval of the column line through $p_{i}$. An index $i$ is called a near-index if the polylines goes through a prime interval containing $p_{i}$ in its closure; otherwise it is called a far-index. For the near-indices, the summation of the errors caused by the new polyline is bounded by $n \delta=\frac{z_{\infty} \varepsilon}{2}$. For each far-index, the errors caused by the new polyline at the column is bounded by $1+\frac{\varepsilon}{2}$ times the one caused by the old (i.e. optimal) polyline. Thus, the combined error of the new polyline for all the far indices is at most $\left(1+\frac{\varepsilon}{2}\right) z_{\mathrm{opt}}$. In total, the error of the new polyline is bounded by $\left(1+\frac{\varepsilon}{2}\right) z_{\mathrm{opt}}+\frac{z_{\infty} \varepsilon}{2} \leq(1+\varepsilon) z_{\mathrm{opt}}$.

Thus, it suffices to compute the optimal tame polyline. There are $M n$ portals, and thus $N=O\left(M^{2} n^{2}\right)$ lines going through a pair of portals. Let $\mathcal{L}$ be the set of these lines. We design a dynamic programming algorithm. For the $i$ th column, for each line $\ell \in \mathcal{L}$ and each $m \leq k$, we record the approximation error of the best $m$-joint tame polyline up to the current column whose (rightmost) link covering $p_{i}$ is on $\ell$. When we proceed to the $(i+1)$ th column, each approximation error is updated. If there is an intersection between lines $\ell$ and $\ell^{\prime}$ in the interval $\left(x_{i}, x_{i+1}\right]$, we consider the polylines that have the intersection as a possible joint. This can be done by copying the data for $\ell$ to $\ell^{\prime}$ and vice versa incrementing the joint number by one, and then keeping the smaller of the current and the new (copied) error for each of the pairs $(\ell, m)$ and $\left(\ell^{\prime}, m\right)$ for $m=1,2, \ldots, k$. Then, we add the distance from $p_{i+1}$ to each polyline. Finally, we select the minimum error at the $n$th column, and retrieve the polyline by backtracking. 
There are $O\left(N^{2}\right)$ intersections of lines, and it takes $O(k)$ time for each intersection for copying and updating. This requires $O\left(N^{2} k\right)$ work and dominates the running time. Since $N=O\left(n^{2} M^{2}\right)=O\left(n^{2} \varepsilon^{-2} \log ^{2}\left(n+\varepsilon^{-1}\right)\right)$, we have the following:

Theorem 4.4. $A(1+\varepsilon)$-approximation, i.e., a $k$-joint polyline with error $1+\varepsilon$ times the optimal, for each of the $L_{1}$ and $L_{2} k$-joint problems can be computed in $O\left(k n^{4} \varepsilon^{-4} \log ^{4}\left(n+\varepsilon^{-1}\right)\right)$ time.

\section{Concluding remarks}

A major open problem is to determine the complexity class of the $k$-joint problem for $L_{1^{-}}$and $L_{2}$-fitting. The corresponding $L_{1}$ or $L_{2}$ polyline approximation problem where the input is a curve is also interesting.

We remark that the curve simplification problem under the $L_{1}$-measure is to minimize the area between input and output polylines. In the restricted case where the vertex set or the set of lines supporting edges of the output polyline is required to be a subset of that of the input polyline, the problem is reduced to the $k$-link shortest path problem in a graph. In particular, if the input polyline is convex, this problem is related to matrix searching (see [2]). However, for the general case the authors are not aware of an efficient algorithm, and it is an interesting research problem. A related question for which polynomial-time algorithms have been constructed for $L_{1}$ and $L_{2}$ measures is approximating an $x$-monotone curve by a $k$-peak curve, i.e., a curve with at most $k$ local maxima [6]. The $k$-joint problem seems to be more difficult than the $k$-peak problem because controlling continuity is a challenge in the former case.

Acknowledgment: The authors would like to thank Jirí Matoušek for a stimulating discussion on convexity.

\section{References}

[1] P. K. Agarwal, S. Hal-Peled, N.H. Mustafa, and Y. Wang, "Near-linear time approximation algorithm for curve simplification," Proc. 2002 European Symp. Algorithms, LNCS 2461, (2002) pp.29-41.

[2] A. Aggarwal, B. Schieber, and T. Tokuyama, "Finding a minimum-weight $k$-link path in graphs with the concave Monge property and applications," Discrete Comput. Geom., 12 (1994) 263-280.

[3] P. Agarwal, B. Aronov, T. Chan, M. Sharir, "On levels in arrangements of lines, segments, planes, and triangles," Discrete Comput. Geom., 19 (1998) 315-331.

[4] P. Agarwal and J. Matoušek, "Ray shooting and parametric search," SIAM J. Comput., 22 (1993) 794-806.

[5] P. K. Agarwal and K. R. Varadarajan, "Efficient algorithms for approximating polygonal chains," Discrete Comput. Geom., 23 (2000) 273-291.

[6] J. Chun, K. Sadakane, and T. Tokuyama, "Linear time algorithm for approximating a curve by a single-peaked curve," Proc. 14th Internat. Symp. Algorithms Comput. (ISAAC 2003), LNCS 2906, 2003, pp. 6-16.

[7] H. Edelsbrunner, Algorithms in Combinatorial Geometry, ETACS Monographs on Theoretical Computer Science 10, Springer-Verlag, 1987.

[8] D. Eu and G.T. Toussaint, "On approximating polygonal curves in two and three dimensions," CVGIP: Graphical Models And Image processing 56 (1994) 231-246.

[9] K. L. Clarkson and P. W. Shor, "Application of random sampling in computational geometry," Discrete Comput. Geom., 4 (1989) 423-432.

[10] M. Goodrich, "Efficient piecewise-linear function approximation using the uniform metric," Discrete Comput. Geom., 14 (1995) 445-462. 
[11] S. Hakimi and E. Schmeichel, "Fitting polygonal functions to a set of points in the plane," Graphical Models and Image Processing, 53 (1991) 132-136.

[12] H. Imai and M. Iri: "Polygonal approximations of a curve - Formulations and algorithms," Computational Morphology, Elsevier Science Publishers B.V. (North Holland), 1988, 71-86.

[13] H. Imai, K. Kato, and P. Yamamoto: "A linear-time algorithm for linear $L_{1}$ approximation of points," Algorithmica, 4 (1989) 77-96.

[14] N. Katoh and T. Tokuyama, "Notes on computing peaks in k-levels and parametric spanning trees," Proc. 17th ACM Symp. on Computational Geometry, 2001, pp. 241-248.

[15] Y. Kurozumi and W.A. Davis: "Polygonal approximation by the minimax method," Computer Graphics and Image Processing, 19 (1982) 248-264.

[16] S. Langerman and W. Steiger, "Optimization in arrangements." Proc. Symp. Theor. Aspects Computer Science (STACS2003), LNCS 2607, 2003, pp. 50-61.

[17] J. Matoušek, "Efficient partition trees," Discrete Comput. Geom., 8 (1992) 315-334.

[18] J. Matoušek, "Range searching with efficient hierarchical cutting," Proc. 8th ACM Symp. on Comput. Geom., (1992) 276-287.

[19] J. Matoušek, "Geometric range searching," ACM Computing Surveys, 26 (1994) 421-461.

[20] J. Matoušek and O. Schwarzkopf, "Linear optimization queries," Proc. 8th Annual ACM Symp. Comput. Geom., 1992, pp. 16-25.

[21] N. Megiddo and A. Tamir, "Finding least-distances lines," SIAM J. Algebraic Discrete Methods, 4 (1983) pp. 207-211.

[22] A. Melkman and J. O'Rourke, "On polygonal chain approximation," Computational Morphology, Elsevier Science Publishers B.V. (North Holland), 1988, 87-95.

[23] J. O'Rourke and G. Toussaint, "Pattern recognition," Chapter 43 of Handbook of Discrete and Computational Geometry (eds. J. Goodman and J. O'Rourke), CRC Press, 1997.

[24] F. P. Preparata and M. I. Shamos, Computational Geometry, an Introduction, Springer-Verlag, New York, 1985.

[25] J. Salowe, "Parametric search," Chapter 37 of Handbook of Discrete and Computational Geometry (eds. J. Goodman and J. O'Rourke), CRC Press, 1997.

[26] T. Tokuyama, "Minimax parametric optimization problems and multi-dimensional parametric searching," Proc. 33rd Symp. Theory Comput. (2001), pp. 75-83.

[27] D. P. Wang, N. F. Huang, H. S. Chao, and R. C. T. Lee, "Plane sweep algorithms for polygonal approximation problems with applications," Proc. 4th Internat. Symp. Algorithms Comput. (ISAAC 2003), LNCS 762, 1993, pp. 515-522.

[28] Robert Weibel, "Generalization of spatial data: principles and selected algorithms," Algorithmic Foundations of Geographic Information Systems, LNCS 1340, 1997, pp. 99-152. 